\title{
JT Format (ISO 14306) and AP 242 (ISO 10303): The Step to the Next Generation Collaborative Product Creation
}

\author{
Alfred Katzenbach ${ }^{1}$, Sebastian Handschuh ${ }^{2}$, and Steven Vettermann ${ }^{3}$ \\ ${ }^{1}$ Executive Consultant \\ alfred.katzenbach@daimler.com \\ ${ }^{2}$ Head of JT Workflow Forum \\ sebastian.handschuh@daimler.com \\ ${ }^{3}$ General Manager of ProSTEP Association \\ steven.vettermann@prostep.com
}

\begin{abstract}
JT format became ISO Standard ISO14306 in December 2012 [1]. It is the beginning of new effective CAD collaboration in Multi-CAD environments. In parallel it gives innovative IT providers the chance to contribute with advanced solutions to challenge the established IT vendors. Through smart combination with the upcoming STEP AP 242 XML for the first time a process-oriented solution for supporting automotive data exchange requirements is available.
\end{abstract}

Keywords: JT Format, ISO 14306, STEP AP 242, ISO 10303, Engineering Collaboration, Product Creation.

\section{$1 \quad$ Introduction}

Digital Engineering Visualization is an approach which gives a broad number of individuals, being part of the product creation processes, the access to 3D data without using a traditional CAD system. As depicted in Figure 1, the number of people who only need to view information is 10 times higher than of its creators.

Over the last 5 to 8 years, JT Format (at this time owned by Siemens PLM) became more and more a de facto standard for $3 \mathrm{D}$ visualization in the automotive and aerospace industry. Hence, the Global Automotive Advisory Group, a forum of the international heads of automotive engineering IT, recommended Siemens PLM to handover the JT file format description to ISO for international standardization. To make this happen, ProSTEP iViP Association, the well-known organization for standardization in the field of engineering collaboration took over the operational lead to provide all documents and arrangements to support the ISO organization in the formal process to get ISO 14306 approved.

The JT format contents not only a definition of a tessellated representation with dedicated level of detail. It defines also an exact geometry description based on BREP and e.g. product and manufacturing information. 


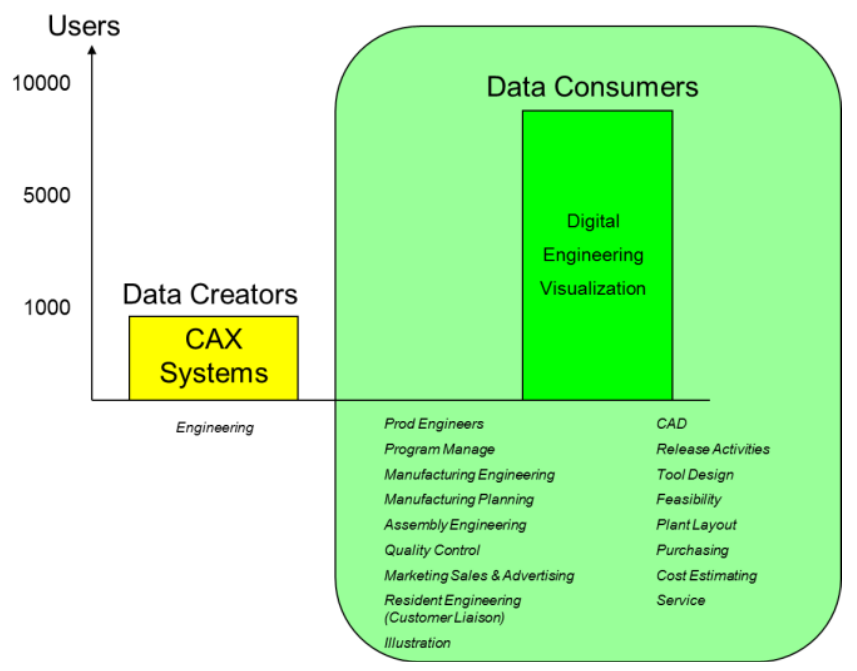

Fig. 1. Figure 1 - Hugh need for viewing information [1]

But for supporting all industrial visualization requirements JT itself could reach its full potential only by applying it in combined application with the smart XML-functionalities of the new STEP AP 242. Thus, in 2010, the decision was taken to establish STEP AP 242 XML and JT as the standard-combination for enabling lightweight data exchange. STEP AP 242 XML, as the process backbone, for assembly, meta, kinematic data etc. And JT as lightweight visualization format for 3D industrial data. By this, for the first time a process-oriented solution for supporting automotive data exchange requirements was agreed.

\section{JT Based Processes}

\subsection{Process Overview}

Only to provide an international standard without defining, how this standard can be used gains only limited value. So it is crucial to define processes first and underline these processes by concrete use cases.

Figure 2 gives an overview of the possible work share of native CAD and JT in an international automotive enterprise. Here we have to distinguish between internal company value adding processes which still require native CAD representation and integration processes between the OEM and the various numbers of suppliers. For this integration tasks, JT can contribute to be more flexible, faster and especially cost efficient. 


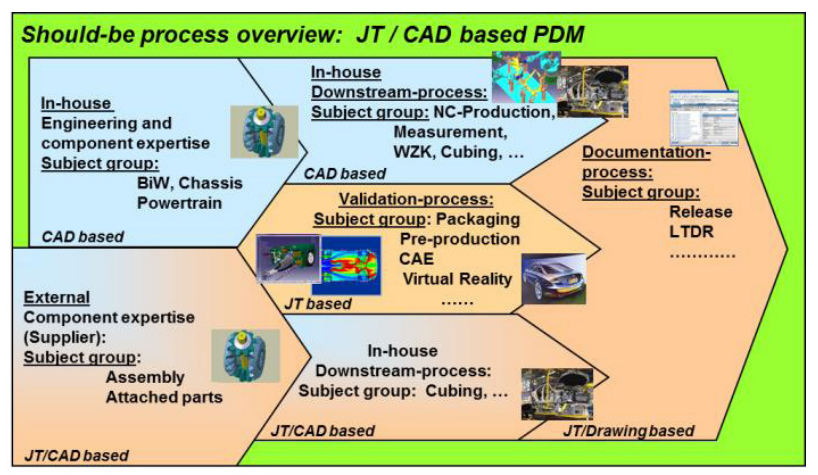

Fig. 2. Figure 2 -Future work share between CAD and JT (Source: Daimler, cf. [3])

\subsection{JT Use Cases}

To go into more detail it is recommended to detail the usage of JT in dedicated workflow descriptions.

The JT Workflow Forum, a working group under the umbrella of Pro STEP iViP Association defined such scenarios and published it in a whitepaper (cf. [3]).

The different use cases were classified in three categories, related to the complexity of usage

- Viewing

- Digital Mock Up

- Design in Context

\subsubsection{Viewing}

Viewing was the foundation of using JT. This includes also high end visualization, bidding and inquiry processes as well as material specification.

\subsubsection{Digital Mock Up}

Beside DMU analysis, the use cases in this cluster define processes for tolerance analysis, drawingless manufacturing, tolerance studies, supplier integration and al processes of digital factory.

\subsubsection{Design in Context}

Here the more advanced processes are defined. This includes hybrid design in context, all simulation activities as long term data retention. 


\section{Desgin in Context Using JT}

\subsection{Hybrid and Non-hybrid Design in Context}

The Design in Context $(\mathrm{DiC})$ process is one of the most complex processes which could be based on JT- and structure information (in future e.g. STEP AP 242XML) coming from various $3 \mathrm{D}-\mathrm{CAD}$-systems. These formats can be combined with native 3D-CAD-data which is often needed and beneficial in design processes (no media break). It is to be distinguished into [7]:

- Hybrid- (HDiC)and

- Non-hybrid design in context (NHDiC).

The hybrid design in context process directly uses JT-/ structure data as an input format without data translation into native 3D-CAD-formats (cf. Figure3).

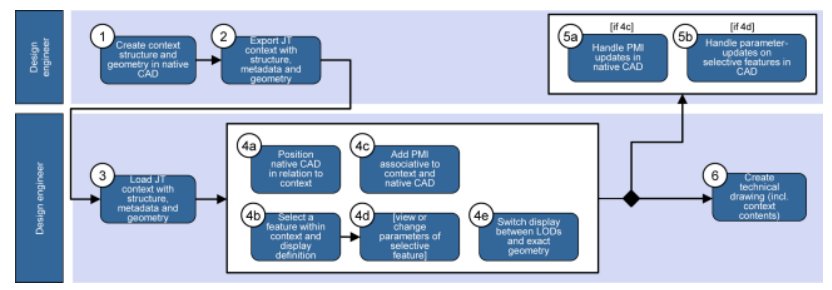

Fig. 3. Hybrid design in context use case [3]

In today's design in context processes often the exchange of geometrical change information is needed. The design in context process often refers to interface regions of (e.g. externally created) parts. Information located in these regions (e.g. geometrical elements) can be used for DiC-processes. The main categories in which this information can be divided are:

- Geometrical- and

- Technological information

Geoemtrical information is often used to define geometrical elements in surrounding parts on customer/OEM side. Therefore information such as diameters, curves or supplemental geometry is used. In case technological information is needed a broad range of information is thinkable in interface regions of parts e.g. mechanical-, electrical-information [7]. These interface related feature information can be transfered out of the sourcing 3D-CAD-system into JT-data using e.g. Product Manufacturing Information (PMI) or in future using a combination of JT and XML-based accompanying dataformats such as STEP AP242XML for the transfer of additional information. Work on this topic was done e.g. at the University of Kaiserslautern in context of value extracting use of open lightweight data formats (such as JT) in automotive collaboration and development process chains [7], [8]. Due to this fact the next chapter gives an overview of the value added chain in automotive industry and describes (as an example) a JT-based data exchange pilot project. 


\section{Supplier Integration}

\subsection{JT Based Supply Chains in Automotive Industry}

The value added in many industries is decreasing on OEM side and increasing on supplier side. Several studies record this (cf.Figure 4) [5],[6]

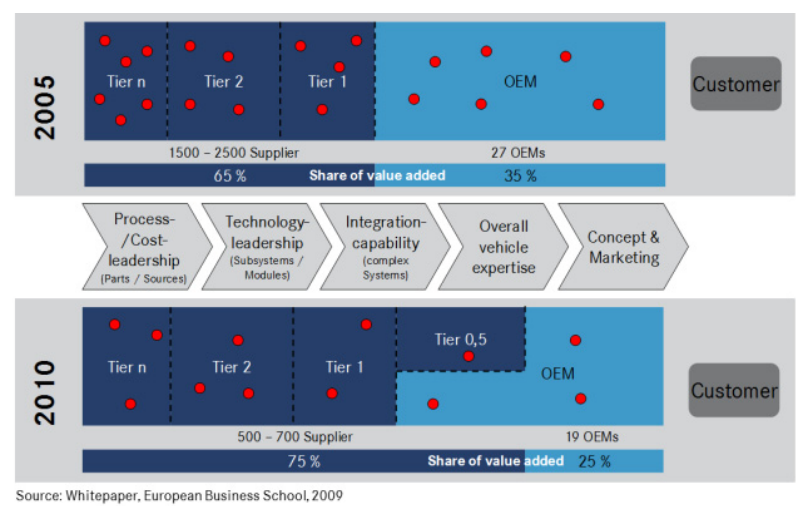

Fig. 4. Value added on OEM/Supplier side [5]

In the automotive industry often suppliers deliver complete car moduls such as door panels or dash boards.

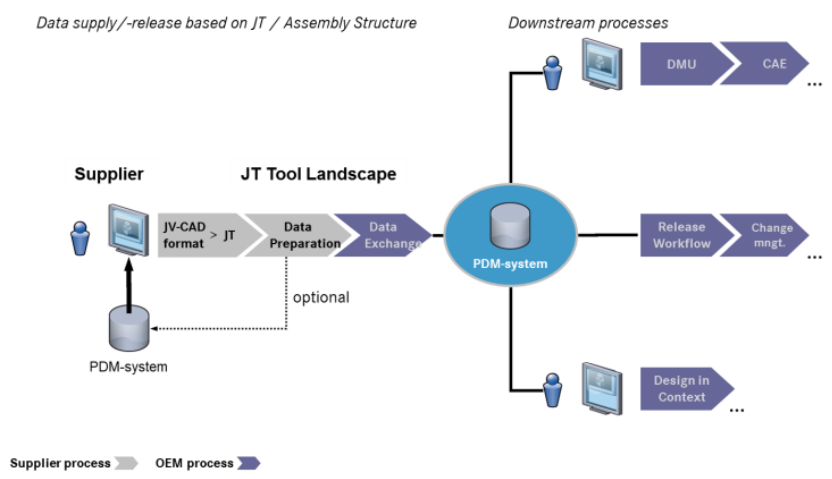

Fig. 5. Big Picture JT-based process land-scape

While system landscapes in context of global sourcing processes are typically heterogeneous the usage of ISO standardized data formats (JT /STEP APA242) for data exchange is getting more and more important. Figure 5 gives an overview of the processlandscape in such supplier integration scenarios. These processes are validated in automotive industry in several pilot projects e.g. with global automotive suppliers (cf. Figure 6). 


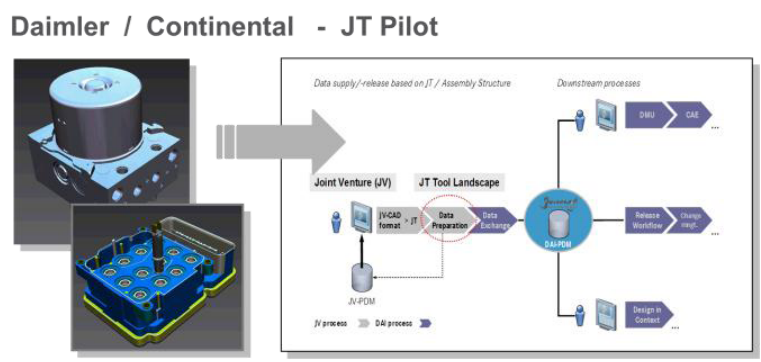

Fig. 6. JT-Pilot project (exchange of an ECU)

The results of these JT-based data exchange processes are very promising and further potentials in using JT instead of native 3D-CAD-data in collaboration processes are identified e.g. in the fields of data-preparation,-handling and -storage. To reach such a duo of complemented and ISO standardized data formats a lot of work has to be done. In the next chapter some detail of the standardization activities are shown.

\section{JT and Step AP 242 Basis for Process-Oriented Data Exchange}

\subsection{Following Modern Standardization Concepts}

Modern IT standards must be flexible and fulfil the industrial requirements. The challenge is to figure out the $20 \%$ that are needed to cover the $80 \%$ of practical applications (Pareto principle).

Standards can only provide industry with the necessary value added if there is a stable basis on which additional functionality can be developed to respond to contextspecific needs. This may take the form of new developments or, preferably, developments that are already in widespread use but which can now be simply transferred to other areas. It should not be necessary to continuously reinvent the wheel. Within the context of Multi-CAD environments this has been seriously addressed by starting the standardization JT and STEP AP 242.

\subsection{STEP Forms the Backbone}

In 2010 representatives of leading aerospace and automotive companies as well as standardization organization came together in order to discuss measures for strengthen the future position of STEP. By this time, with regard to 3D engineering the two major standards "ISO 10303-203 - Configuration controlled 3D design of mechanical parts and assemblies" and "ISO 10303-214 - Core data for automotive mechanical design processes" reached an overlap of functionality by $75 \%$ over the years (cf. Figure 2). This was regarded as costly and a dissemination hindrance. Thus, the merger of these two standards was decided: STEP AP 242 (ISO 10303-24 - Managed model based $3 d$ engineering). 
AP 242 is designed to address the common aerospace and automotive use cases and to provide a single data backbone. Its publication as ISO standard is planned for beginning of 2014.

AP 242 offers more and/or enhanced functionalities compared with its parents AP's, AP 203 and AP 214. But it also offers additionally a new concept: the Business Object Model, the so-called BO-Model.

Major enhancements are provided in AP 242 by adding functionalities for (cf. Figure 3):

- Composites

- Kinematics

- Product Manufacturing Information

- Rules and Requirements

- Shape Data Quality

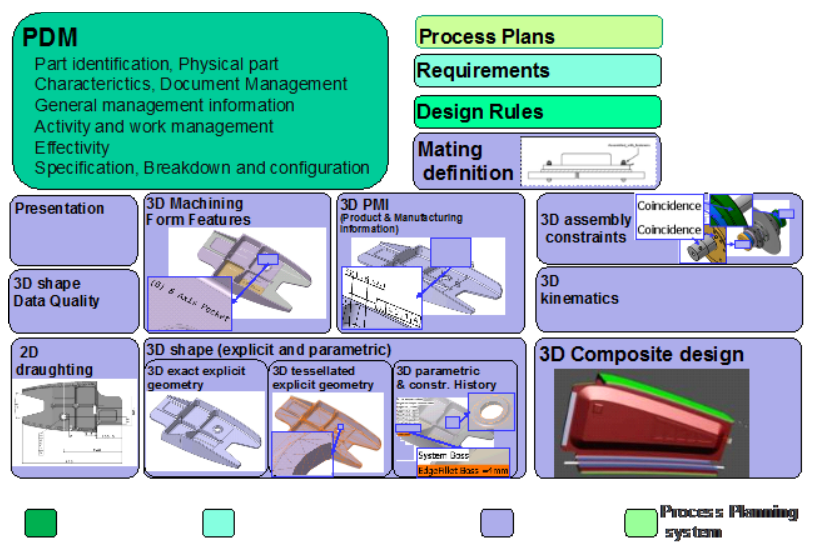

Fig. 7. Figure 3: AP 242 information model overview

Additionally, the new External Element Reference Mechanism is integral part of AP 242. Unlike in the past, by this mechanism it is not only possible to reference a file (e.g. from an assembly tree to parts), it is now possible to reference elements within a (STEP)file (e.g. a curve, an edge, an axis). This is extremely important for e.g. kinematics, where one needs to reference certain constraints.

The BO-Model is a subset of the AP 242 functionalities. It covers all data relevant for data management, e.g. meta data. This also means, that it does not cover e.g. 3D geometry itself, it only links to it. Beside the tradition representation of a STEP-File via a STEP Physical File (SPF), the BO-Model also provides a representation expressing the equivalent information in XML. Thus, the intent behind the BO-Model is to provide a standard-based representation of product data management data, as SPF and in XML. 
Especially the new capabilities to express PDM-relevant data in XML and the described External Element Reference Mechanism make AP 242 so attractive to use it together with JT in the field of visualization. And this was one of the main motivations to go for this.

\subsection{JT as the Leafs of a (STEP)tree}

In the past, STEP became the standard for the exchange of product model data. There is no better. But in one field it failed: visualization.

One could now state: So, let's invest resources and in some years we could close this gap. But in 2010 industry required a standard 'right now'.

In the same time, STEP matured to what it is today, JT matured to the leading visualization solution. Thus, it was obvious to start the standardization of JT via ISO and to take care, that the two standards could by applied separately, but if they are used together, that they form a process-supporting solution which is more powerful than its pieces.

In 2010, the decision was taken to propose the standardization of the complete JT Version 9.5 via ISO, so that independent and royalty-free solutions can be realized. Figure 4 show the harmonized roadmap between the two standardization projects JT and STEP AP 242.

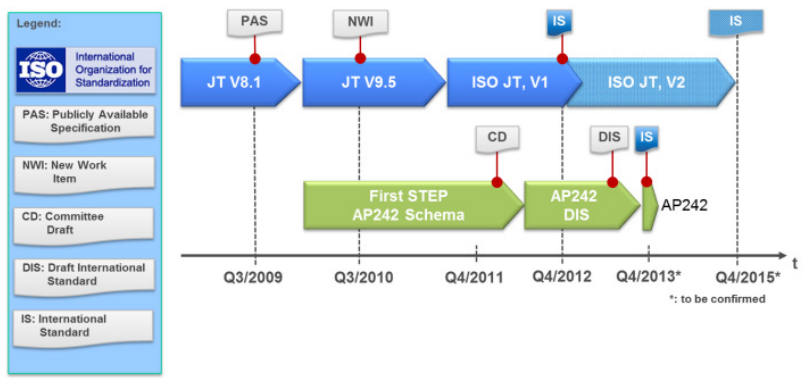

Fig. 8. Figure 4: ISO Roadmap for JT and STEP AP 242

The agreed aim was to establish a visualization standard which could use the enhanced XML-functionalities of STEP AP 242. By this, JT should be kept lightweight. Frankly said, JT provides the 3D-relevant data within a STEP-tree.

\subsection{JT Data Model}

The data model for JT includes more than just geometry models (cf. Figure 5). On the one hand, it offers the option of displaying the models in various degrees of precision and, on the other hand, other information that is important for product development and downstream processes can be stored together with the model. 

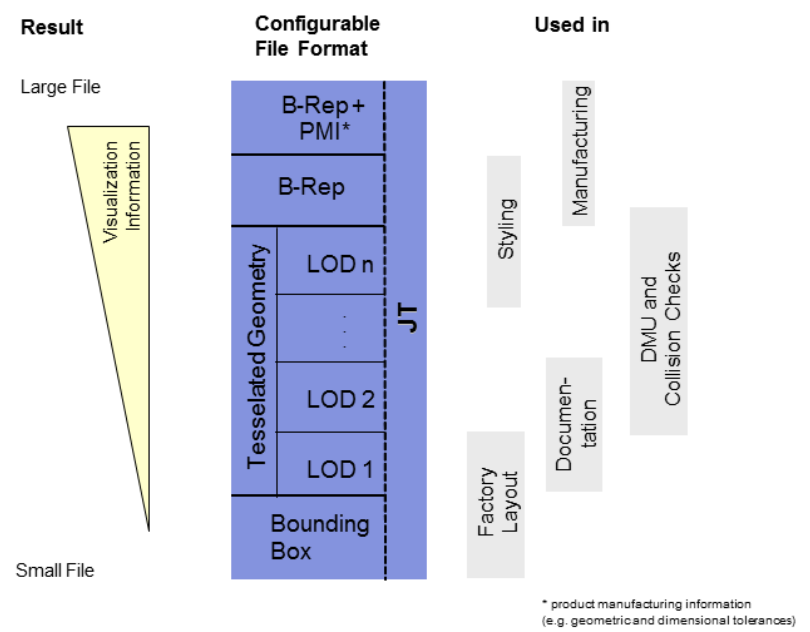

Fig. 9. JT Data Model

In addition to the ability to display geometry, it was the fact that product manufacturing information (PMI) such as tolerances, toleranced dimensions and information about surface properties could be displayed together with the $3 \mathrm{D}$ models and processed further that contributed to its fast proliferation.

\section{Geometry}

- At one of the lowest levels, simple regular geometry such as cuboids, cylinders and pyramids - for the definition of designed spaces for example - are located in what is referred to as the bounding box.

- Tessellated or faceted geometry allows the representation of solids and surfaces. JT offers the option of defining different levels of detail (LOD), i.e. specifying how precisely something is to be represented. A very low LOD means less precision and a smaller volume of data, a very high LOD means a level of precision approaching the exact geometry and a larger volume of data.

- The latest form of compression is JT ULP, which was released in 2008 and allows the volume of data to be reduced to almost one hundredth the size of the original data.

- BREP (boundary representation) geometry offers the highest level of precision. They are an exact representation of the solids and surfaces that were generated in the CAD system, for example. The exact JT file is also only a fraction of the size of the original file.

Through certain compression algorithms it's possible to reduce the size of a representing the same content tremendously (cf. Figure 6). 


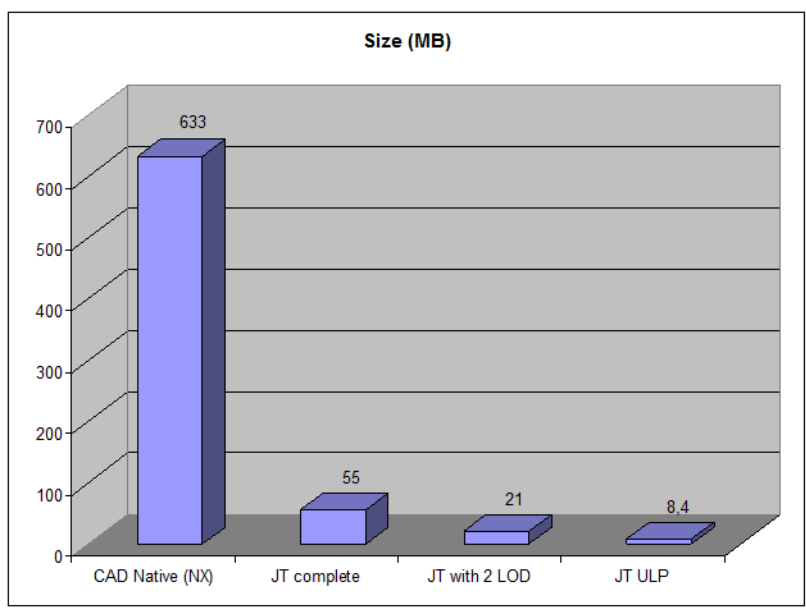

Fig. 10. Figure 6: JT file size comparison (Source: Siemens PLM)

\section{Opportunities for Innovative It Provider}

The various application scenarios give rise to numerous requirements that need to be met by the JT and STEP data. Questions also rise with regard to the quality of the JT data supplied by the systems involved. In other words, on the one hand they relate to the authoring systems and on the other hand to the translators that generate JT from a native format or can convert JT into a native format.

In order to provide a reliable answer to these questions, systematic benchmarking of the tools involved is needed. Until now, the ProSTEP iViP Association, together with the VDA PLM Working Group, conducted three neutral Benchmarks. The latest was published beginning of 2013 (cf. [4]). In this benchmark the focus was on LODund PMI-related functionalities as well as the performance of available JT viewers.

Based on the derived use cases and the related requirements that the JT and STEP need to satisfy the JT Workflow Forum, which comprises a number of companies from the automotive industry, and the JT Implementor Forum, which comprises leading IT vendors and providers of translators and system integrations, set up metrics and test models for conducting state-of-the-art benchmarks. A participants gathered rich information out of the fruitful discussions and took the chance for process and product enhancements.

The next benchmark is already in preparation and the complexity as well as the expectations with regard to the performance of the tested products is rising year by year (cf. Figure 7).

The upcoming benchmark will focus on the two building blocks illustrated in Fig. 8. 


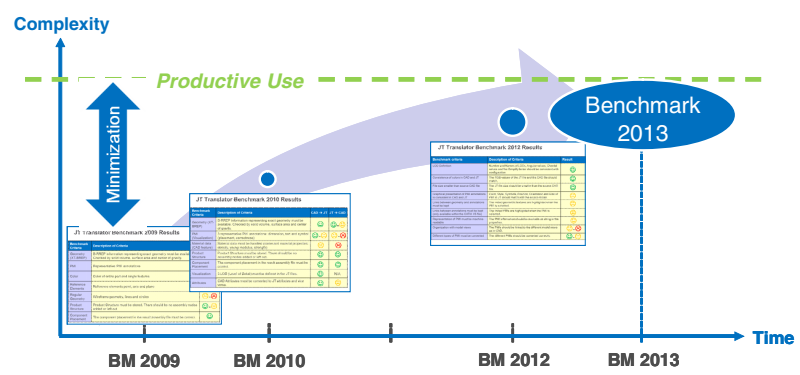

Fig. 11. Complexity of the test scenarios is raising
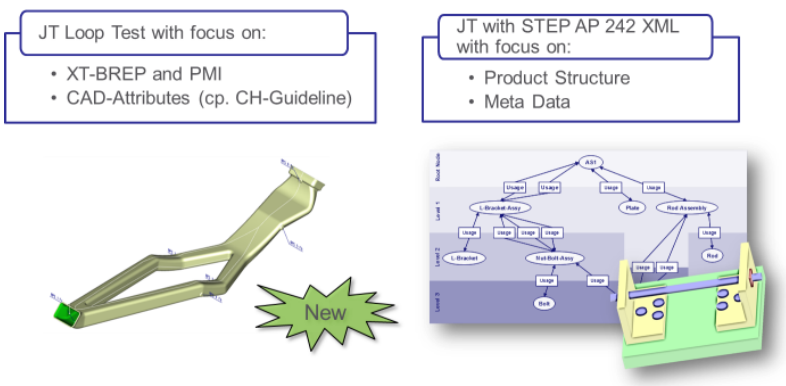

Fig. 12. Building blocks of the JT Benchmark 2013

Speaking about Multi-CAD environments, within these benchmark the involved CAD Systems are CATIA V5, Creo 2, NX 8.5 and Solidworks 2012. And the tested translators are from Core Technologie, Geometrics, Elysium, ITI Transcen-Data, Kronion, Siemens PLM, SpaceClaim, Theorem and T-Systems.

The already gathered information underline that industry took the right step when deciding to address the challenge of Multi-CAD environments with a combination of the standards JT and STEP.

\section{Conclusions}

JT and STEP AP 242 are on the way to change CAD based development and gain much more benefits out of 3D data than possible in the past.

The ISO Standards provide a reliable and sustainable definition for product describing data including product structure and behaviour models.

Powerful low cost and or freeware solutions will appear for a more intensive use of product data. Special solutions for ultra-light weight descriptions under JT definitions are on the way to be established.

For DMU and other integration processes international and multi partner collaboration can be defined in a new way. Here the specific solutions of each partner can be respected as long as he is committed to the two ISO standards. 
Design in context solutions have a better chance when they support JT and AP 242. This will based force the reincarnation of the special CAD solutions for dedicated tasks. Secondly special solutions will be developed directly using JT definition. The one fits all CAD "Dinos" might have a tough future.

In this spirit ProSTEP iViP Association and most of the associated companies are engaged to work on JT Version 2 to enhance to value added position of JT and AP 242.

\section{References}

1. ISO: Industrial automation systems and integration - JT file format specification for 3D visualization, ISO 14306:2012 (2012), http: / / www . iso . org

2. SASIG: D-21 Digital Engineering Visualization (2005), http: / /www . sasig.org

3. ProSTEP iViP: White Paper Applying JT (2010), http: / / www . prostep. org

4. ProSTEP iViP: JT Application Benchmark (2013), http: / /www . prostep org

5. Forschungsgemeinschaft für Außenwirtschaft (FAST), Studie: Innovationsstrategien für erfolgreiche Automobilzulieferer (2008)

6. European Business School, White Paper 2009 (2009)

7. Handschuh, S.: Wertextrahierende Nutzung von offenen leichtgewichtigen Datenformaten in automobilen Kollaborations- und Entwicklungs-prozessketten. Dissertation Technische Universität Kaiserslautern, Schriften-reihe VPE, Kaiserslautern, vol. 11 (2011)

8. Roth, M., Sprickmann, A.: Prozessüber-greifende Nutzung von VR-Technologien im Schiffbau (Tagungsband Go3D). Fraunhofer Institut für Grafische Datenverarbeitung, Rostock (2010) 